\title{
The Value of Preoperative Blood Neutrophil- Lymphocyte, White Blood Cell-Lymphocyte, Monocyte-Lymphocyte, and Platelet-Lymphocyte Ratios in Predicting the Malignant Potential of Gastrointestinal Stromal Tumor: A Retrospective Study
}

Lizhao Yan

Wuhan Union Hospital

Yinghao Cao

Wuhan Union Hospital

Shenghe Deng

Wuhan Union Hospital

Junnan Gu

Wuhan Union Hospital

Fuwei Mao

Wuhan Union Hospital

Yifan Xue

Wuhan Union Hospital

Hang Li

Wuhan Union Hospital

Xinghua Liu ( $\square$ liu_xinghua@hust.edu.cn )

Wuhan Union Hospital https://orcid.org/0000-0001-8783-0573

Research article

Keywords: Gastrointestinal stromal tumour, Neutrophil-lymphocyte rate, White blood cell-lymphocyte rate, Monocyte-lymphocyte rate, Platelet-lymphocyte rate

Posted Date: April 5th, 2021

DOl: https://doi.org/10.21203/rs.3.rs-386992/v1

License: (c) (i) This work is licensed under a Creative Commons Attribution 4.0 International License. Read Full License 
Page 2/15 


\section{Abstract}

Background: To investigate the predictive value of the neutrophil-lymphocyte ratio (NLR), white blood celllymphocyte ratio (WLR), monocyte-lymphocyte ratio (MLR), and platelet-lymphocyte ratio $(P L R)$ in grading the malignant potential of the gastrointestinal stromal tumour (GIST).

Methods: We retrospectively reviewed 843 GIST patients who underwent surgery during hospitalisation from June 2016 to July 2020 and 374 unrelated healthy controls. Demographic data were collected for each participant, including age, gender, body mass index, laboratory test results, and pathological results. We compared the NLR, WLR, MLR, and PLR between GIST and healthy individuals, as well as among patients with different risk grades of GIST.

Results: NLR, WLR, MLR, and PLR values of patients with GIST were significantly increased compared with those of the control group $(P<0.05)$. These values also showed statistically significant differences among the four risk degree groups. The NLR, WLR, MLR, and PLR values of the high-risk group were significantly higher than those of the very low-risk group, low-risk group, and intermediate-risk group $(p<0.001)$. The NLR, WLR, MLR, and PLR showed a high predictive value in the high-risk group, with an area under the receiver operating characteristic curve of $0.802,0.824,0.866$, and 0.814 , and a cut-off value of $2.059,3.347,0.221$, and 141.4 , respectively.

Conclusion: Preoperative NLR, WLR, MLR, and PLR have a high predictive value for grading the malignant potential of GIST, especially in the high-risk group. These values provide a convenient and inexpensive way to determine preoperative chemotherapy and surgical strategies.

\section{Introduction}

Gastrointestinal stromal tumour (GIST) is the most common mesenchymal tumour of the gastrointestinal tract, with an unadjusted annual incidence of approximately 1.5 per $100,000[1]$. The most common primary tumour site is the stomach (40-70\%), followed by the small intestine (20-40\%) and the colorectum (5-15\%), and it can also occasionally occur in the oesophagus, mesentery, omentum, and retroperitoneum[2]. GISTs originate from Cajal cells in the gastrointestinal tract wall, which are the precursors of gastrointestinal stromal cells and are located in the myenteric plexus of the lamina propria[3].

Complete surgical resection remains a standard treatment for primary resectable GIST[4]. The latest American National Comprehensive Cancer Network guidelines indicate that surgery aims to completely excise the GIST without rupturing the tumour capsule and damaging the nearby organs; otherwise, preoperative imatinib mesylate treatment should be considered after the tumour grade and histological subtype have been established with a biopsy[5]. Imatinib, a small molecular inhibitor of receptor tyrosine kinases, is one of the few tumour-targeting drugs successfully applied in clinical practice, and has dramatically changed the paradigm of GIST treatment[6]. Preoperative imatinib can shrink the tumour 
without targeting the surrounding organ, increasing the chance of complete tumour resection without extensive organ resection[7-9].

The National Institutes of Health $(\mathrm{NIH})$ criteria have been widely used by clinicians, which stratify GISTs into four risk degrees classification: "very low-risk," low-risk," "intermediate-risk," and "high-risk" [8]. The $\mathrm{NIH}$ criteria not only provide useful information to predict patient prognosis, but can also determine GIST patients who may benefit from adjuvant therapy[10]. Its predictive value has been evaluated in several large retrospective cohort studies[11]. These criteria depend on pathological findings; however, due to the soft texture of the GIST tumour, an improper preoperative biopsy can lead to implantation metastasis and haemorrhage.

Above all, preoperative grading is of great significance for predicting the prognosis and determining the preoperative chemotherapy strategy. However, due to the risk and cumbersome task of performing a biopsy, it is necessary to find an alternative accurate and convenient method of preoperative grading.

In recent years, the value of cancer-related inflammatory factors in determining patient survival and disease progression has emerged for various types of cancer[12]. Systemic inflammation can promote tumour progression and metastasis by inhibiting apoptosis and promoting angiogenesis. The neutrophillymphocyte ratio (NLR) is an economical, convenient, and repeatable measure that can be used to detect the systemic inflammatory response, and a high NLR is closely related to the prognosis of a variety of tumours, including GIST[13-16]. The peripheral blood cell-lymphocyte ratio (WLR), monocyte-lymphocyte ratio (MLR), and platelet-lymphocyte ratio (PLR) have also been shown to be prognostic factors for various malignant solid tumours[17, 18].

This study aimed to assess the predictive value of preoperative peripheral blood NLR, WLR, MLR, and PLR for classifying the risk of GIST, and compare differences in their predictive value between different risk grades of GIST.

\section{Materials And Methods}

\section{Study design and patient selected}

Patients who underwent resection of a primary GIST without preoperative chemotherapy between 2016 and 2020 were identified from Wuhan Union hospitals. The clinical, surgical, and pathological data of the patients were retrospectively collected from clinical files. The inclusion criteria were as follows: (1) the patient was initially diagnosed as having a primary completely resectable GIST; (2) the patient had complete peripheral blood test results within 7 days before surgery; (3) the patient had complete followup data; and (4) the patient provided informed consent. Patients with the following conditions were excluded from the study: (1) patients who underwent preoperative chemoradiotherapy and targeted therapy with imatinib or its analogues; (2) patients with other cancers; (3) patients with various types of inflammation within 7 days before surgery; and (4) patients with severe cardiopulmonary diseases. The 
study was approved by the ethics committee of Wuhan Union Medical College Hospital (No. 2018-S377), and all patients provided written informed consent to participate.

\section{Definition and classification criteria}

NLR was defined as the ratio of neutrophils to lymphocytes, WLR as the ratio of white blood cells to lymphocytes, MLR as the ratio of monocytes to lymphocytes, and PLR as the ratio of platelets to lymphocytes. According to the NIH criteria for GIST, all patients were classified into very low-risk, low-risk, intermediate-risk, and high-risk based on tumour size, mitotic count, tumour sites, and rupture status.

\section{Statistical analysis}

All statistical analyses were performed using the $\mathrm{R}$ language environment version 3.5.2. Continuous variables were presented as mean \pm standard deviation or median, and categorical variables were expressed as a percentage. Students t-test was performed to compare the difference in continuous variables. After the normal test for each group of data, the data did not follow the normal distribution. Kruskal-Wallis $\mathrm{H}$ test was used for the comparison between multiple groups of data, and Bonfferoni corrected A value that was used for the comparison between the two groups. The ROC curve was drawn with specificity as the abscisic coordinate and sensitivity as the ordinate coordinate. $P$ values less than 0.05 were considered statistically significant.

\section{Results}

\section{Patient characteristics}

A total of 843 GIST patients who underwent surgery during hospitalisation from June 2016 to July 2020 and 374 unrelated healthy controls were included. In the GIST patient, males accounted for $55.16 \%$ of participants, and the average age was 55.17 years. There were 141 cases in the very low-risk group, 265 cases in the low-risk group, 94 cases in the intermediate-risk group, and 343 cases in the high-risk group. Three hundred and seventy-six healthy subjects from Wuhan Union Hospital were included as healthy controls during the same period. There were no statistically significant differences in age, sex, or body mass index between the two groups $(P>0.05)$. Compared with the control group, the NLR, PLR, WLR, and MLR values of GIST patients were significantly increased $(P<0.001)$ (Table 1). Furthermore, the NLR, WLR, MLR, and PLR values of GIST patients of all risk grades were significantly higher than patients in the control group $(P<0.05$, respectively) (Table 2$)$. Upon comparing the different risk groups, the NLR, WLR, MLR, and PLR values in the high-risk group were significantly higher than that in the very low-risk group, low-risk group, and intermediate-risk group $(P<0.05)$. In contrast, there was no statistically significant difference among the very low-risk group, low-risk group, and intermediate-risk group $(P>$ 0.05). 
Table 1

Comparison between experimental group and control group.

\begin{tabular}{|c|c|c|c|c|c|c|}
\hline & \multirow{2}{*}{$\begin{array}{l}\text { Control } \\
\text { group } \\
\left(\begin{array}{l}n=376) \\
(\%)\end{array}\right.\end{array}$} & \multicolumn{4}{|c|}{ Experimental group $(n=843)(\%)$} & \multirow[t]{2}{*}{$\mathbf{P}$} \\
\hline & & $\begin{array}{l}\text { Extra-low } \\
\text { level } \\
(n=141)\end{array}$ & $\begin{array}{l}\text { Low level } \\
(n=265)\end{array}$ & $\begin{array}{l}\text { Medium } \\
\text { level } \\
(n=94)\end{array}$ & $\begin{array}{l}\text { High level } \\
(n=343)\end{array}$ & \\
\hline Sex & & & & & & $>0.05^{a}$ \\
\hline male & 172(45.74) & $64(45.39)$ & $150(56.60)$ & $44(46.81)$ & 207(60.35) & \\
\hline female & $204(54.26)$ & $77(54.61)$ & $115(43.40)$ & $50(53.19)$ & 136(39.65) & \\
\hline Age $($ mean $\pm s d)$ & $\begin{array}{l}56.38 \pm \\
10.86\end{array}$ & $\begin{array}{l}57.19 \pm \\
10.19\end{array}$ & $\begin{array}{l}57.92 \pm \\
10.76\end{array}$ & $\begin{array}{l}59.11 \pm \\
10.75\end{array}$ & $\begin{array}{l}57.44 \pm \\
10.75\end{array}$ & $>0.05^{b}$ \\
\hline BMI & $22.5 \pm 3.06$ & $\begin{array}{l}23.5 \pm \\
2.77\end{array}$ & $22.5 \pm 1.62$ & $23.4 \pm 2.18$ & $22.1 \pm 2.36$ & $0.695^{b}$ \\
\hline $\begin{array}{l}\text { PLR (medium } \pm \\
\text { qrange) }\end{array}$ & $\begin{array}{l}103.88 \pm \\
39.85\end{array}$ & $\begin{array}{l}124.50 \pm \\
69.79\end{array}$ & $\begin{array}{l}144.00 \pm \\
89.23\end{array}$ & $\begin{array}{l}147.84 \pm \\
71.45\end{array}$ & $\begin{array}{l}170.40 \pm \\
128.94\end{array}$ & $\begin{array}{l}< \\
0.0001^{c}\end{array}$ \\
\hline $\begin{array}{l}\text { WLR (medium } \pm \\
\text { qrange) }\end{array}$ & $2.84 \pm 0.82$ & $\begin{array}{l}3.17 \pm \\
1.10\end{array}$ & $3.54 \pm 1.81$ & $3.56 \pm 1.96$ & $4.03 \pm 2.50$ & $\begin{array}{l}< \\
0.0001^{\mathrm{c}}\end{array}$ \\
\hline $\begin{array}{l}\text { MLR (medium } \pm \\
\text { qrange) }\end{array}$ & $0.16 \pm 0.07$ & $\begin{array}{l}0.22 \pm \\
0.13\end{array}$ & $0.25 \pm 0.15$ & $0.25 \pm 0.21$ & $0.29 \pm 0.21$ & $\begin{array}{l}< \\
0.0001^{c}\end{array}$ \\
\hline $\begin{array}{l}\text { NLR (medium } \pm \\
\text { qrange) }\end{array}$ & $1.60 \pm 0.75$ & $\begin{array}{l}1.85 \pm \\
1.00\end{array}$ & $2.14 \pm 1.67$ & $2.19 \pm 1.71$ & $2.62 \pm 2.32$ & $\begin{array}{l}< \\
0.0001^{c}\end{array}$ \\
\hline
\end{tabular}


Table 2

Different risk groups were compared with the control group.

\begin{tabular}{|c|c|c|c|c|c|c|c|c|c|}
\hline & $\begin{array}{l}\text { Control } \\
\text { group }\end{array}$ & $\begin{array}{l}\text { Extra- } \\
\text { low } \\
\text { level }\end{array}$ & $\begin{array}{l}\text { Low } \\
\text { level }\end{array}$ & $\begin{array}{l}\text { Medium } \\
\text { level }\end{array}$ & $\begin{array}{l}\text { High } \\
\text { level }\end{array}$ & P1 & P2 & P3 & P4 \\
\hline $\begin{array}{l}\text { PLR } \\
\text { (medium } \pm \\
\text { qrange) }\end{array}$ & $\begin{array}{l}103.88 \\
\pm 39.85\end{array}$ & $\begin{array}{l}124.50 \\
\pm 69.79\end{array}$ & $\begin{array}{l}144.00 \\
\pm 89.23\end{array}$ & $\begin{array}{l}147.84 \\
\pm 71.45\end{array}$ & $\begin{array}{l}170.40 \\
\pm 128.94\end{array}$ & $\begin{array}{l}<.05 \\
0.05\end{array}$ & $\begin{array}{l}<.05 \\
0.05\end{array}$ & $\begin{array}{l}<.05 \\
0.05\end{array}$ & $\begin{array}{l}<.05 \\
0.05\end{array}$ \\
\hline $\begin{array}{l}\text { WLR } \\
\text { (medium } \pm \\
\text { qrange) }\end{array}$ & $\begin{array}{l}2.84 \pm \\
0.82\end{array}$ & $\begin{array}{l}3.17 \pm \\
1.10\end{array}$ & $\begin{array}{l}3.54 \pm \\
1.81\end{array}$ & $\begin{array}{l}3.56 \pm \\
1.96\end{array}$ & $\begin{array}{l}4.03 \pm \\
2.50\end{array}$ & $\begin{array}{l}<.05 \\
0.05\end{array}$ & $\begin{array}{l}<.05 \\
0.05\end{array}$ & $\begin{array}{l}<.05 \\
0.05\end{array}$ & $<.05$ \\
\hline $\begin{array}{l}\text { MLR } \\
\text { (medium } \pm \\
\text { qrange) }\end{array}$ & $\begin{array}{l}0.16 \pm \\
0.07\end{array}$ & $\begin{array}{l}0.22 \pm \\
0.13\end{array}$ & $\begin{array}{l}0.25 \pm \\
0.15\end{array}$ & $\begin{array}{l}0.25 \pm \\
0.21\end{array}$ & $\begin{array}{l}0.29 \pm \\
0.21\end{array}$ & $\begin{array}{l}<.05 \\
0.05\end{array}$ & $\begin{array}{l}<.05 \\
0.05\end{array}$ & $\begin{array}{l}< \\
0.05\end{array}$ & $\begin{array}{l}<.05 \\
0.05\end{array}$ \\
\hline $\begin{array}{l}\text { NLR } \\
\text { (medium } \pm \\
\text { qrange) }\end{array}$ & $\begin{array}{l}1.60 \pm \\
0.75\end{array}$ & $\begin{array}{l}1.85 \pm \\
1.00\end{array}$ & $\begin{array}{l}2.14 \pm \\
1.67\end{array}$ & $\begin{array}{l}2.19 \pm \\
1.71\end{array}$ & $\begin{array}{l}2.62 \pm \\
2.32\end{array}$ & $\begin{array}{l}<.05 \\
0.05\end{array}$ & $\begin{array}{l}<.05 \\
0.05\end{array}$ & $\begin{array}{l}< \\
0.05\end{array}$ & $\begin{array}{l}<.05 \\
0.05\end{array}$ \\
\hline \multicolumn{10}{|c|}{$P$ value is calculated by the Dunnett's $t$ test. } \\
\hline \multicolumn{10}{|c|}{ P1: between control group and extra-low level group; } \\
\hline \multicolumn{10}{|c|}{ P2: between control group and low level group; } \\
\hline \multicolumn{10}{|c|}{ P3: between control group and medium level group; } \\
\hline
\end{tabular}

\section{Receiver operating characteristic curve analysis in the very low-risk group}

According to the receiver operating characteristic curve (ROC), the MLR was found to have a relatively good predictive power in the very low-risk, low-risk, and intermediate-risk groups. However, the NLR, WLR, and PLR were found to have a relatively poorer predictive power in the very low-risk, low-risk, and intermediate-risk groups (Table 3). 
Table 3

The cut-off values between different risk grou

\begin{tabular}{|c|c|c|c|c|c|c|}
\hline & Danger & Area & $95 \% \mathrm{Cl}$ & Cut point & Sensitive & Specific \\
\hline \multirow[t]{4}{*}{ NLR } & Extra-low level & 0.603 & $(0.5452,0.6606)$ & 1.727 & 0.603 & 0.582 \\
\hline & Low level & 0.693 & $(0.6504,0.7357)$ & 2.033 & 0.543 & 0.769 \\
\hline & Medium level & 0.698 & $(0.6296,0.7655)$ & 2.008 & 0.617 & 0.747 \\
\hline & High level & 0.802 & $(0.7704,0.8340)$ & 2.059 & 0.673 & 0.782 \\
\hline \multirow[t]{4}{*}{ WLR } & Extra-low level & 0.623 & $(0.5662,0.6805)$ & 3.375 & 0.426 & 0.790 \\
\hline & Low level & 0.713 & $(0.6715,0.7546)$ & 3.348 & 0.566 & 0.782 \\
\hline & Medium level & 0.716 & $(0.6498,0.7817)$ & 3.306 & 0.649 & 0.755 \\
\hline & High level & 0.824 & $(0.7937,0.8534)$ & 3.347 & 0.708 & 0.782 \\
\hline \multirow[t]{4}{*}{ MLR } & Extra-low level & 0.739 & $(0.6872,0.7917)$ & 0.182 & 0.709 & 0.678 \\
\hline & Low level & 0.792 & $(0.7551,0.8285)$ & 0.185 & 0.755 & 0.705 \\
\hline & Medium level & 0.772 & $(0.7111,0.8319)$ & 0.243 & 0.543 & 0.915 \\
\hline & High level & 0.866 & $(0.8392,0.8936)$ & 0.221 & 0.749 & 0.843 \\
\hline \multirow[t]{4}{*}{ PLR } & Extra-low level & 0.660 & $(0.6019,0.7178)$ & 138.7 & 0.461 & 0.854 \\
\hline & Low level & 0.719 & $(0.6767,0.7613)$ & 144.0 & 0.502 & 0.880 \\
\hline & Medium level & 0.757 & $(0.6938,0.8194)$ & 131.8 & 0.638 & 0.795 \\
\hline & High level & 0.814 & $(0.7812,0.8464)$ & 141.4 & 0.665 & 0.867 \\
\hline
\end{tabular}

\section{ROC curve analysis in the low-risk group}

The MLR was found to have relatively good predictive power in the low-risk group (AUROC: 0.792 [95\% CI 0.7551-0.8285], sensitivity: 75.5\%, specificity: 70.5\%, P < 0.01). However, the NLR (AUROC: 0.693 [95\% Cl: 0.6504-0.7357], sensitivity: 54.3\%, specificity: 76.9\%, P<0.01), WLR (AUROC: 0.713 [95\% Cl: 0.67150.7546], sensitivity: 56.6\%, specificity: 78.2\%, P< 0.01), and PLR (AUROC: 0.719 [95\% Cl: 0.6767-0.7613], sensitivity: $50.2 \%$, specificity: $88.0 \%, P<0.01$ ) were found to have relatively poorer predictive power (Table 3).

\section{ROC curve analysis in the intermediate-risk group}

The MLR was found to have relatively good predictive power in the intermediate-risk group (AUROC: 0.772 [95\% Cl: 0.7111-0.8319], sensitivity: 54.3\%, specificity: 91.5\%, P < 0.01). However, the NLR(AUROC: 0.698 [95\% Cl 0.6296-0.7655], sensitivity: 54.3\%, specificity: 76.9\%, P< 0.01), WLR (AUROC: 0.716 [95\% Cl: 0.6498-0.7817], sensitivity: $64.9 \%$, specificity: $75.5 \%$, P < 0.01), and PLR (AUROC: 0.757 [95\% Cl: 0.6938- 
0.8194], sensitivity: $63.8 \%$, specificity: $79.5 \%, \mathrm{P}<0.01)$ were found to have relatively poorer predictive power.

\section{ROC curve analysis in the high-risk group}

All four indicators showed the best predictive power in the high-risk group, with the MLR (AUROC 0.866 [95\% Cl: 0.8392-0.8936], sensitivity: 74.9\%, specificity: $84.3 \%, \mathrm{P}<0.01$ ) being the most prominent. The NLR (AUROC: 0.802 [95\% Cl: 0.7704-0.8340], sensitivity: 67.3\%, specificity: 78.2\%, $P<0.01$ ), WLR (AUROC: 0.824 [95\% Cl: 0.7937-0.8534], sensitivity: 70.8\%, specificity: 78.2\%, $\mathrm{P}<0.01$ ), and PLR (AUROC: 0.814 [95\% Cl: 0.7812-0.8464], sensitivity: $66.5 \%$, specificity: $86.7 \%, \mathrm{P}<0.01$ ) also showed relatively good predictive power. The cut-off values for the NLR, WLR, MLR, and PLR were 2.059, 3.347, 0.221, and 141.4 , respectively.

\section{Discussion}

With the recent improvements in the risk grading criteria, and the in-depth clinical study of imatinib, the diagnosis and treatment of GISTs has dramatically improved. The risk criteria proposed by the NIH are widely used worldwide and was shown to have a reliable correlation with patient survival after GIST surgery[8, 19]. Imatinib is one of the few tumour-targeting drugs with significant clinical efficacy that has been incorporated into the standard postoperative treatment regimen for patients with high-risk GIST. Preoperatively, imatinib can shrink the tumour to promote the complete surgical resection and reduce the occurrence of surgical complications, especially for local high-risk GIST[20]. A precise classification of the risk grade can have a significant impact on surgery and prognosis. However, the classification criteria rely on pathological results, so it is important to accurately predict and classify the risk grade before surgery.

PLR, WLR, MLR, and NLR are biomarkers of the immune response, and have been shown to be prognostic factors for many types of tumours. East et al.[17] found that the NLR and WLR were independent predictors of postoperative overall survival in colorectal cancer. For GIST, Racz et al.[15] showed that a high PLR is related to a poor prognosis in GIST patients. Goh et al.[16] found that a high PLR and high NLR are independent risk factors for postoperative progression-free survival in GIST patients. Cananzi et al.[18] found that a high MLR was negatively correlated with disease-free survival after GIST surgery. Peripheral blood cell detection is a convenient and routinely performed preoperative test, but only a few studies have explored the predictive value of inflammatory biomarkers for GIST risk grading.

In the present study, we evaluated the value of inflammatory markers such as PLR, WLR, MLR, and NLR in the risk grading of GISTs, compared the differences in their predictive value between different GIST risk groups, and evaluated their diagnostic significance. We found that the PLR, WLR, MLR, and NLR had no obvious diagnostic significance in the very low-risk group, but showed good predictive power in the lowand medium-risk groups. In contrast, the diagnostic significance in the high-risk group was the most prominent. At present, many studies have assessed the prognostic significance of the NLR and PLR for GISTs. A high NLR and PLR are often associated with poor prognosis, which is consistent with our 
results. The cut-off values of NLR and PLR (2.059 and 131.8) showed a good predictive power in the high-risk group. Few studies have assessed the prognostic significance of the WLR in GISTs. Therefore, we first evaluated the predictive significance of the WLR for grading the risk of GISTs, and found that this had a higher predictive value compared with the NLR and PLR. The MLR has been reported as a prognostic factor in studies involving colorectal cancer, multiple myeloma, Hodgkin's lymphoma, and other types of tumours, and has also been confirmed as an independent prognostic factor for diseasefree survival after GIST surgery in the study by Cananzi[18]. In this study, the MLR showed a better predictive value in the intermediate-risk and low-risk groups, and also exhibited the most valuable predictive value compared with other inflammatory markers in the high-risk group.

This study has some limitations. Firstly, this was a retrospective study. Although promising results were obtained, there are still few studies on the MLR and WLR for GIST, and further prospective studies are needed to verify these results. Secondly, there are numerous grading criteria for GIST, and we only evaluated the widely used $\mathrm{NIH}$ standards, so the practicability of this study was limited.

In summary, this study assessed a large sample of 843 patients and found that preoperative PLR, WLR, $M L R$, and NLR values were all good predictors for the risk of GIST; however, there were significant differences in the predictive value among different risk groups. In the very low-risk group, the predictive value of all four markers was deficient, but these markers showed better predictive value in the low-risk and intermediate-risk groups. However, these four markers had the best predictive value in the high-risk group, among which, the MLR was the most evident. Clinically, the peripheral blood test is a cheap and convenient method that can help determine the risk grade of GIST before surgery, and can provide a reliable basis for the selection of targeted preoperative imatinib therapy and postoperative survival evaluation.

\section{Conclusions}

Preoperative NLR, WLR, MLR, and PLR have a high predictive value for classifying the different risk grade of GIST, especially in the high-risk group, which can be used as an effective indicator in predicting malignant potential of GIST before treatment.

\section{Abbreviations}

NLR

Neutrophil-lymphocyte ratio

WLR

White blood cell-lymphocyte ratio

MLR

Monocyte-lymphocyte ratio

PLR

Platelet-lymphocyte ratio 
GIST

Gastrointestinal stromal tumour

ROC

Receiver operating characteristic curve

HR

Hazard ratio

$\mathrm{Cl}$

Confidence interval

\section{Declarations}

\section{Author contributions}

L.Y, and Y.C lead the study. Y.C. performed the data analysis, implemented the methodology; S.H., J.G., F.M., Y.X., collected the data; L.Y, and Y.C. prepared the original draft; Y.C. and H.L. helped to perfect the figures. X.L. reviewed and edited the final manuscript.

\section{Funding}

This research was funded by the Graduates' Innovation Fund, Huazhong University of Science and Technology (No. 2020yjsCXCY055, 2020yjsCXCY080); Free innovation pre-research fund and platform scientific research fund in 2019 (02.03.2019-111).

\section{Availability of data and materials}

The datasets used and/or analysed during the current study are available from the corresponding author on reasonable request.

\section{Ethics approval and consent to participate}

The study ethics approval was granted from the local ethical committee of the Wuhan Union Hospital (No.2018-S377), and the study was performed in accordance with the principles of the Declaration of Helsinki.

\section{Consent for publication}

Not applicable.

\section{Competing interests}

The authors declare that they have no competing interests.

\section{References}


1. Miettinen M, Lasota J: Gastrointestinal stromal tumors. Gastroenterol Clin North Am 2013, 42(2):399415.

2. Suehara Y, Akaike K, Mukaihara K, Kurisaki-Arakawa A, Kubota D, Okubo T, Mitomi H, Mitani K, Takahashi M, Toda-lshii M et al: KCTD12 is negatively regulated by Kit in gastrointestinal stromal tumors. Oncotarget 2018, 9(43):27016-27026.

3. Serrano-Candelas E, Ainsua-Enrich E, Navines-Ferrer A, Rodrigues P, Garcia-Valverde A, Bazzocco S, Macaya I, Arribas J, Serrano C, Sayos $\mathrm{J}$ et al: Silencing of adaptor protein SH3BP2 reduces KIT/PDGFRA receptors expression and impairs gastrointestinal stromal tumors growth. $\mathrm{Mol}$ Oncol 2018, 12(8):1383-1397.

4. Hemming ML, Lawlor MA, Zeid R, Lesluyes T, Fletcher JA, Raut CP, Sicinska ET, Chibon F, Armstrong SA, Demetri GD et al: Gastrointestinal stromal tumor enhancers support a transcription factor network predictive of clinical outcome. Proc Natl Acad Sci U S A 2018, 115(25):E5746-E5755.

5. von Mehren M, Randall RL, Benjamin RS, Boles S, Bui MM, Ganjoo KN, George S, Gonzalez RJ, Heslin MJ, Kane JM et al: Soft Tissue Sarcoma, Version 2.2018, NCCN Clinical Practice Guidelines in Oncology. J Natl Compr Canc Netw 2018, 16(5):536-563.

6. Mazzocca A, Napolitano A, Silletta M, Spalato Ceruso M, Santini D, Tonini G, Vincenzi B: New frontiers in the medical management of gastrointestinal stromal tumours. Ther Adv Med Oncol 2019, 11:1758835919841946.

7. Chang WL, Yang WC, Zeng XY, Li CG, Xiong Z, Wang T, Zhang RZ, Tao KX, Zhang P: Elevated preoperative platelet-to-lymphocyte ratio predicts poor prognosis of patients with primary gastrointestinal stromal tumor. BMC Gastroenterol 2020, 20(1):124.

8. Joensuu H: Risk stratification of patients diagnosed with gastrointestinal stromal tumor. Hum Pathol 2008, 39(10):1411-1419.

9. Joensuu H, Eriksson M, Sundby Hall K, Reichardt A, Hermes B, Schutte J, Cameron S, Hohenberger P, Jost PJ, Al-Batran SE et al: Survival Outcomes Associated With 3 Years vs 1 Year of Adjuvant Imatinib for Patients With High-Risk Gastrointestinal Stromal Tumors: An Analysis of a Randomized Clinical Trial After 10-Year Follow-up. JAMA Onco/ 2020.

10. Nishida T: Asian consensus guidelines for gastrointestinal stromal tumor: what is the same and what is different from global guidelines. Trans/ Gastroenterol Hepatol 2018, 3:11.

11. von Mehren M, Joensuu H: Gastrointestinal Stromal Tumors. J Clin Oncol 2018, 36(2):136-143.

12. Reinehr T, Roth CL: Inflammation Markers in Type 2 Diabetes and the Metabolic Syndrome in the Pediatric Population. Curr Diab Rep 2018, 18(12):131.

13. Kargin S, Cakir M, Gundes E, Yavuz Y, Esen HH, Sinan lyisoy M, Kokbudak N, Kucukkartallar T: Relationship of preoperative neutrophil lymphocyte ratio with prognosis in gastrointestinal stromal tumors. Ulus Cerrahi Derg 2015, 31(2):61-64.

14. Rutkowski P, Gronchi A, Hohenberger P, Bonvalot S, Schoffski P, Bauer S, Fumagalli E, Nyckowski P, Nguyen BP, Kerst JM et al: Neoadjuvant imatinib in locally advanced gastrointestinal stromal tumors (GIST): the EORTC STBSG experience. Ann Surg Oncol 2013, 20(9):2937-2943. 
15. Racz JM, Cleghorn MC, Jimenez MC, Atenafu EG, Jackson TD, Okrainec A, Venkat Raghavan L, Quereshy FA: Predictive Ability of Blood Neutrophil-to-Lymphocyte and Platelet-to-Lymphocyte Ratios in Gastrointestinal Stromal Tumors. Ann Surg Oncol 2015, 22(7):2343-2350.

16. Goh BK, Chok AY, Allen JC, Jr., Quek R, Teo MC, Chow PK, Chung AY, Ong HS, Wong WK: Blood neutrophil-to-lymphocyte and platelet-to-lymphocyte ratios are independent prognostic factors for surgically resected gastrointestinal stromal tumors. Surgery 2016, 159(4):1146-1156.

17. East JM, Hogan J, Samaha G, Medani M, MacKerricher W, Polinkevych S, Walsh SR, Coffey JC: Ratios derived from an array of standard haematological indices predict the oncological outcome in colon cancer. Colorectal Dis 2014, 16(6):442-449.

18. Cananzi FCM, Ruspi L, Quagliuolo VL: Preoperative monocyte-to-lymphocyte ratio predicts recurrence in gastrointestinal stromal tumors. J Surg Oncol 2019, 119(7):1026.

19. Wei Z, Wu J, Huang W, Li J, Lu X, Yuan Y, Xiong W, Zhang X, Wang W, He Y et al: Immune-infiltration based signature as a novel prognostic biomarker in gastrointestinal stromal tumour. 2020, 57:102850.

20. Tirumani SH, Jagannathan JP, Krajewski KM, Shinagare AB, Jacene H, Ramaiya NH: Imatinib and beyond in gastrointestinal stromal tumors: A radiologist's perspective. AJR Am J Roentgeno/ 2013, 201(4):801-810.

\section{Figures}



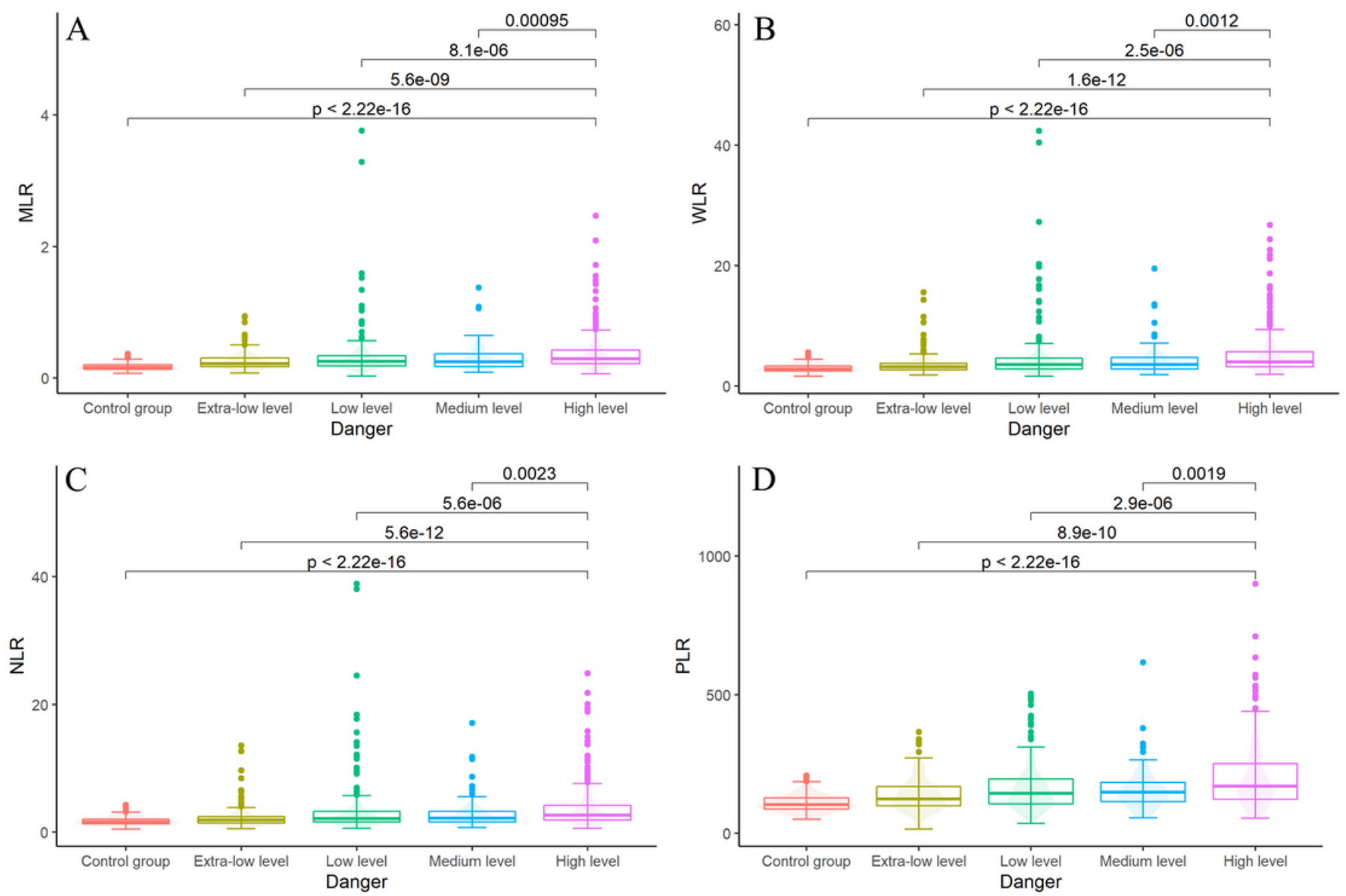

\section{Figure 1}

Comparison of MLR, WLR, NLR, and PLR between different risk groups. A: Comparison of MLR between different risk groups; B: Comparison of WLR between different risk groups; C: Comparison of NLR between different risk groups; D: Comparison of PLR between different risk groups. 


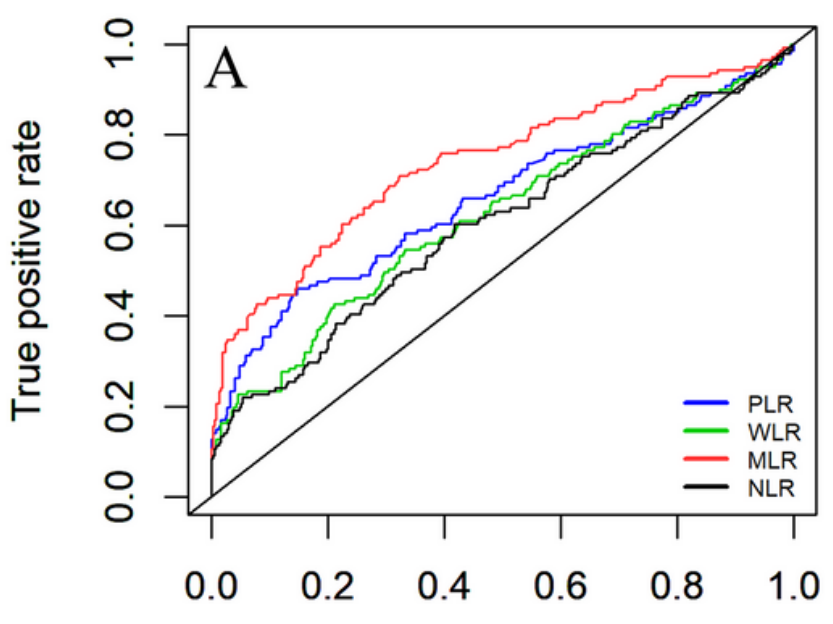

False positive rate

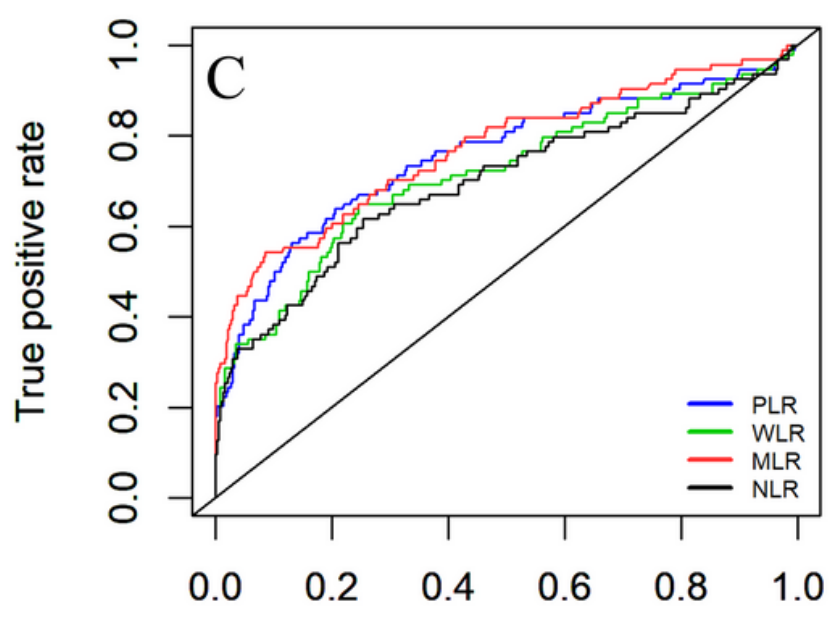

False positive rate

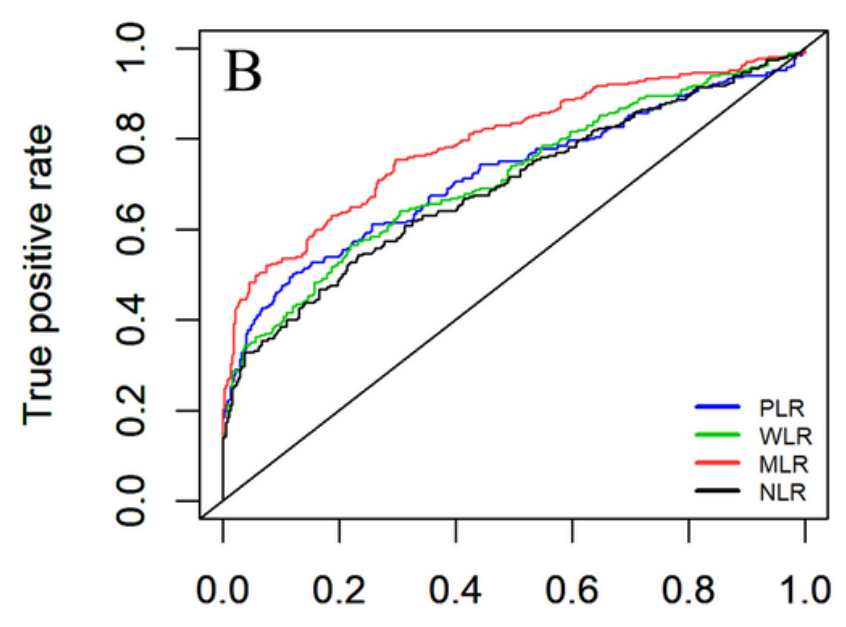

False positive rate

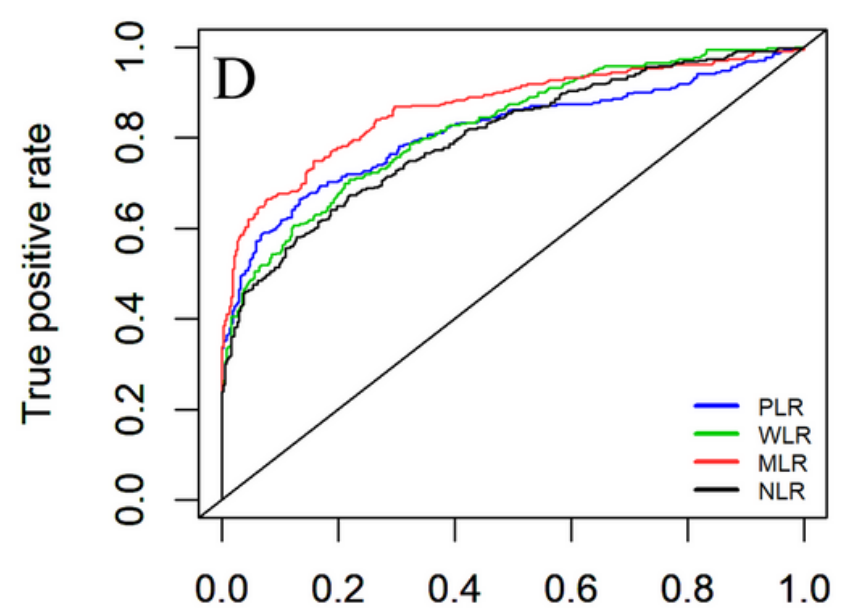

False positive rate

Figure 2

ROC curve analysis in the different risk group. A: Receiver operating characteristic curve analysis in the very low-risk group; B: ROC curve analysis in the low-risk group; C: ROC curve analysis in the intermediaterisk group; D: ROC curve analysis in the high-risk group. 